\title{
Programmed cell death 2 protein induces gastric cancer cell growth arrest at the early $S$ phase of the cell cycle and apoptosis in a p53-dependent manner
}

\author{
JIAN ZHANG, WEI WEI, HUI-CHENG JIN, RONG-CHAO YING, \\ A-KAO ZHU and FANG-JIE ZHANG \\ Department of Gastroenterological Surgery, Hangzhou First People's Hospital, School of Clinical Medicine, \\ Nanjing Medical University, Hangzhou 310006, P.R. China
}

Received May 29, 2014; Accepted August 2, 2014

DOI: 10.3892/or.2014.3551

\begin{abstract}
Programmed cell death 2 (PDCD2) is a highly conserved nuclear protein, and aberrant PDCD2 expression alters cell apoptosis. The present study aimed to investigate PDCD2 expression in gastric cancer. Tissue specimens from 34 gastric cancer patients were collected for analysis of PDCD2 expression using immunohistochemistry, western blotting and qRT-PCR. Gastric cancer cell lines (a p53-mutated MKN28 line and a wild-type p53 MKN45 line) were used to assess the effects of PDCD2 overexpression. $p 53^{-/-}$nude mice were used to investigate the effect of PDCD2 on ultraviolet B (UVB)induced skin carcinogenesis. The data showed that PDCD2 expression was reduced in gastric cancer tissue specimens, and loss of PDCD2 expression was associated with the poor survival of patients. PDCD2 expression induced gastric cancer cell growth arrest at the early $\mathrm{S}$ phase of the cell cycle and apoptosis. The antitumor effects of PDCD2 expression were dependent on $\mathrm{p} 53$ expression in gastric cancer cells. Moreover, PDCD2 expression inhibited activity of the ATM/Chk1/2/p53 signaling pathway. In addition, PDCD2 expression suppressed UVB-induced skin carcinogenesis in $p 53^{+/+}$nude mice, but not in $p 53^{-/}$mice. The data from the present study demonstrated that loss of PDCD2 expression could contribute to gastric cancer development and progression and that PDCD2-induced gastric cancer cell growth arrest at the early $S$ phase of the cell cycle and apoptosis are p53-dependent.
\end{abstract}

\section{Introduction}

Gastric cancer is a significant worldwide health problem and accounts for more than 900,000 new cases of cancer annually

Correspondence to: Dr Rong-Chao Ying, Department of Gastroenterological Surgery, Hangzhou First People's Hospital, School of Clinical Medicine, Nanjing Medical University, Hangzhou 310006, P.R. China

E-mail: zjhz1994@yeah.net

Key words: PDCD2, p53, apoptosis, cell cycle, gastric cancer and caused $\sim 740,000$ cancer-related deaths in 2008 worldwide (1). Although the exact etiology of gastric cancer remains to be defined, multiple genetic and epigenetic alterations, such as activation of oncogenes, silencing of tumor-suppressor genes and genetic instability, are implicated in a multistep process of gastric cancer development (2). Thus, further studies on the gastric carcinogenesis process could support the development of novel strategies for effectively controlling gastric cancer in future clinical practice.

Towards this end, programmed cell death 2 protein (PDCD2) has attracted attention since this protein was originally identified during the apoptosis of rat thymocytes (3). Human $P D C D 2$ is located at chromosome 6q27, a region that is involved in both translocations and deletions in leukemia and lymphoma $(4,5)$. PDCD2 plays a critical role during embryonic development; for example, a mutant of Drosophila PDCD2 (i.e., Zfrp8) resulted in embryonic developmental delay, larval and pupal lethality $(4,6)$. Knockout mouse data indicate that PDCD2 is essential for embryonic stem cell viability and self-renewal (7) and is enriched in three types of mouse stem cells (embryonic, neural and hematopoietic) (8) and in human embryonic stem cells compared to their differentiated derivatives (9). Thus, PDCD2 is thought to be a potential auxiliary factor in stem cell maintenance and differentiation. Alteration of PDCD2 expression could contribute to human cancer development and progression. Fan et al (10) showed that PDCD2 expression is decreased in multidrug-resistant colon cancer cells. However, subsequent studies failed to associate PDCD2 expression with tumor cell apoptosis $(5,6)$. Thus, in the present study, we first analyzed the expression of PDCD protein and mRNA in gastric cancer and matched normal tissues. We next investigated the antitumor effects of PDCD2 on gastric cancer cell lines and the underlying molecular events by confirmation of p53-dependent, PDCD2-mediated apoptosis in gastric cancer cells. We also utilized p53-knockout mice as an in vivo ultraviolet light (UV)-induced skin carcinogenesis model to confirm the role of p53 in mediating the effects of PDCD2 on the regulation of cell apoptosis. The objective of the present study was to provide novel insight into the molecular mechanisms of PDCD2's action in gastric tumorigenesis. 


\section{Materials and methods}

Tissue specimens. Tissue specimens were obtained from 34 patients without pre-surgical chemotherapy or radiotherapy at the Department of Gastroenterological Surgery, Hangzhou First Hospital, School of Clinical Medicine, Nanjing Medical University between January 2009 and December 2013. The study was approved by our hospital review board, and each patient provided informed consent. Fresh tissue specimens and paraffin blocks were used to assess gene expression.

$q R T$-PCR. Total cellular RNA was isolated from tissue samples or cells using an RNeasy Mini kit (Biomed, Beijing, China) and reversely transcribed into first-strand cDNA using a Takara reverse transcription kit (Takara, Dalian, China) and oligo (dT)15 primers (Takara) according to the manufacturer's instructions. The resultant cDNA was then used for qPCR amplification of PDCD2 and p53 expression, and GAPDH mRNA was used as a control. PDCD2 primers were 5'-CTGTGGAGCTGGGCTTCGCC-3' and 5'-CAGCAGGAAGGAGAGCGGGC-3'. p53 primers were 5'-ACTCCAGCCACCTGTAGTCCAAAAAGGGTC-3' and 5'-GACCCTTTTTGGACTACAGGTGGCTGGAGT-3'. GAPDH primers were 5'-AGAAGGCTGGGGCTCATTTG-3' and 5'-AGGGGCCATCCACAGTCTTC-3'. Amplification of $P D C D 2, p 53$ and $G A D P H$ mRNA was performed with one cycle at $95^{\circ} \mathrm{C}$ for $10 \mathrm{~min}$ and 40 cycles of $95^{\circ} \mathrm{C}$ for $15 \mathrm{sec}$ and $60^{\circ} \mathrm{C}$ for $60 \mathrm{sec}$. Calculation of the relative expression of each transcript was performed using the $2^{-\Delta \Delta \mathrm{Ct}}$ method.

Protein extraction and western blotting. Tissues and cells were lysed in a lysis buffer (20 mM Tris- $\mathrm{HCl}, 150 \mathrm{mM} \mathrm{NaCl}$, $2 \mathrm{mM}$ EDTA and $1 \%$ Triton-X100) containing a protease inhibitor cocktail (Sigma-Aldrich, St. Louis, MO, USA). Cellular protein was then quantified using the BCA protein assay kit (Beyotime, Beijing, China). Equivalent amounts of protein samples $(20 \mu \mathrm{g})$ were separated using $12 \%$ sodium dodecyl sulfate (SDS)-polyacrylamide gel electrophoresis (PAGE) and transferred to polyvinylidene fluoride (PVDF) membranes (Millipore Corp., Billerica, MA, USA). Anti-PDCD2 (sc-377250) and anti- $\beta$-actin (sc-130301) antibodies were purchased from Santa Cruz Biotechnology (Santa Cruz, CA, USA), and cell cycle checkpoint-regulated proteins, including anti-ataxia telangiectasia mutated (ATM), anti-p53, anti-checkpoint kinase (Chk)1 and anti-Chk2 were purchased from Santa Cruz Biotechnology. Anti-phospho-S345-Chk1 and anti-phospho-T68-Chk2 antibodies were from Cell Signaling Technology (Danvers, MA, USA). Each specific antibody binding was detected with horseradish peroxidase (HRP)-conjugated respective secondary antibodies (Amersham Biosciences, Amersham, UK) and enhanced chemiluminescence (ECL) solutions (Amersham Biosciences).

Immunohistochemistry. Tumor tissues were fixed in $4 \%$ paraformaldehyde for $24 \mathrm{~h}$, embedded in paraffin and then sectioned into $4-\mu \mathrm{m}$ sections for immunohistochemistry. Briefly, endogenous peroxidase activity was blocked by incubating sections in $3 \%$ hydrogen peroxide for $30 \mathrm{~min}$. Antigen retrieval was performed in citrate buffer $(10 \mathrm{mM}, \mathrm{pH} 6.0)$ for $30 \mathrm{~min}$ at $95^{\circ} \mathrm{C}$ in a pressure cooker. After that, the sections were blocked in
$1.5 \%$ blocking serum in phosphate-buffered saline (PBS) for $2 \mathrm{~h}$ at room temperature and incubated with an anti-PDCD2 antibody (1:200 dilution) or anti-p53 antibody (1:200 dilution; both from Santa Cruz Biotechnology) overnight at $4^{\circ} \mathrm{C}$ in a moist chamber. The next day sections were washed with PBS and incubated with a biotinylated secondary antibody at $37^{\circ} \mathrm{C}$ for $2 \mathrm{~h}$ before exposure to a streptavidin complex (HRP; Beyotime). Positive reaction was visualized using 3,3'-diaminobenzidine tetrahydrochloride (DAB; Beyotime) followed by counterstaining with hematoxylin (Beyotime). The stained sections were reviewed and scored independently under a light microscope by two researchers. Positive staining was defined as $25 \%$ or more of tumor cells staining positively.

Cell lines and culture. Gastric cancer MKN28 cells (p53 mutant) and MKN45 cells (p53 wild-type) were obtained from the American Type Culture Collection (ATCC; Manassas, VA, USA) and grown in RPMI-1640 medium (HyClone, Logan, UT, USA) supplemented with $10 \%$ fetal bovine serum (FBS; Invitrogen, Carlsbad, CA, USA) and antibiotics $(100 \mathrm{U} / \mathrm{ml}$ penicillin and $100 \mu \mathrm{g} / \mathrm{ml}$ streptomycin) in a humidified incubator with $5 \% \mathrm{CO}_{2}$ at $37^{\circ} \mathrm{C}$.

Gene transfection. Plasmids carrying PDCD2 or p53 cDNA, namely pCDNA-3.1-PDCD2 or pCDNA-3.1-p53, respectively, were obtained from Dr Cong Jie (China Medical University). p53 shRNA (cat \# sc-29435) plasmid was obtained from Santa Cruz Biotechnology. For gene transfection, gastric cancer MKN28 and MKN45 cells were grown overnight and transfected with pCDNA-3.1-PDCD2 using Lipofectamine 2000 (Invitrogen) according to the manufacturer's instructions. Plasmid pCDNA-3.1-p53 was transfected into MKN28-PDCD2 cells and $p 53$ shRNA was transfected into MKN45-PDCD2 cells using Lipofectamine 2000.

$\left[{ }^{3} \mathrm{H}\right]$ thymidine incorporation assay. [Methyl $\left.-{ }^{3} \mathrm{H}\right]$ thymidine was obtained from Sigma-Aldrich. Cells (1,000 cells/well) following gene transfection were seeded at least in quadruplicate in 96-well plates, grown for 4 days and then incubated with $20 \mathrm{mCi} /$ well [methyl- ${ }^{3} \mathrm{H}$ ] thymidine for $16 \mathrm{~h}$. Subsequently, the cells were washed with ice-cold PBS and lysed with ice-cold $10 \%$ trichloroacetic acid (TCA) in PBS for $20 \mathrm{~min}$ at $4^{\circ} \mathrm{C}$. Ice-cold $0.2 \mathrm{M} \mathrm{NaOH}$ was then added to the cells and incubated for $10 \mathrm{~min}$ at $-20^{\circ} \mathrm{C}$. The cell lysates were then collected, universal scintillation cocktail $(2.5 \mathrm{ml})$ was added and the incorporation of $\left[{ }^{3} \mathrm{H}\right]$ thymidine into DNA was assessed using a liquid scintillation counter (Xi'an Nuclear Instrument Factory, Xi'an, China) to determine the disintegrations per minute $(\mathrm{dpm})$ values.

Flow cytometric cell cycle and apoptosis assays. To detect cell cycle distribution, the cells following $48 \mathrm{~h}$ of gene transfection were harvested, suspended in PBS and fixed with 70\% ethanol at $4^{\circ} \mathrm{C}$. After centrifugation (1,500 $\mathrm{x}$ g for $\left.5 \mathrm{~min}\right)$, the supernatants were discarded, cellular DNA was stained with $10 \mu \mathrm{M}$ propidium iodide (PI; Keygen, Nanjing, China) and samples were analyzed by a FACSCalibur flow cytometer (BD Biosciences, Baltimore, MD, USA).

Apoptosis was determined using an apoptosis detection kit (Keygen). Briefly, cells following $48 \mathrm{~h}$ of gene transfection 
were collected, washed twice in ice-cold PBS and then resuspended in binding buffer at a density of $1 \times 10^{6}$ cells $/ \mathrm{ml}$. The cells were simultaneously incubated with fluorescein-labeled Annexin V and PI for $20 \mathrm{~min}$. The mixture was then analyzed using a FACSCalibur (BD Biosciences).

Labeling of cells with thymidine analogs. Actively replicating cells at the beginning of each hour of the $S$ phase were first labeled with the thymidine analog, 5-iodo-2'-deoxyuridine (IdU; $50 \mu \mathrm{M}$, Sigma-Aldrich) for $40 \mathrm{~min}$, washed three times with PBS and then labeled with 5-chloro-2'-deoxyuridine (CldU; $100 \mu \mathrm{M}$, Sigma-Aldrich) for $40 \mathrm{~min}$. The IdU and CldU incorporated into replicating DNA were then detected with red or green fluorescent antibodies, respectively. Specifically, slides were treated with $70 \%$ ethanol, washed in PBS, denatured in $2.5 \mathrm{M} \mathrm{HCl}$ for $30 \mathrm{~min}$, permeabilized in $0.25 \%$ Triton $\mathrm{X}-100$ for $5 \mathrm{~min}$ and blocked with $1 \%$ bovine serum albumin. The slides were incubated at room temperature with the following antibodies: i) a mouse anti-bromodeoxyuridine antibody at a dilution of 1:500 (detects IdU; Sigma-Aldrich); ii) an AlexaFluor 488-conjugated anti-mouse antibody at a dilution of 1:1,000 (Invitrogen); iii) a rat anti-bromodeoxyuridine antibody at a dilution of 1:2,000 (detects CldU; Santa Cruz Biotechnology) and iv) an AlexaFluor 633-conjugated anti-rat antibody at a dilution of 1:1,000 (Invitrogen). After counterstaining with DAPI ( $1 \mu \mathrm{g} / \mathrm{ml}$, KeyGen), images were captured under an Olympus CX71 fluorescence microscope (Olympus, Tokyo, Japan).

UVB-induced skin carcinogenesis in p53/- and $p 53^{+/+}$nude mice. The animal study was approved by the ethics committee of our University. According to a previous study (11), male haired $p 53^{-/}$mice with a C57BL/6J genetic background from the Jackson Laboratory (Bar Harbor, ME, USA) were mated with female hairless SKH-1 mice ( $p 53$ wild-type) to obtain male and female hairless congenic p53-deficient mice. The hairless $p 53^{-/}$mice were intercrossed to obtain homozygous p53-deficient mice and their wild-type littermates. Then, UV lamps (FS72T12-UVB-HO, National Biological Corp., Twinsburg, OH, USA) were used to expose $30 \mathrm{p}^{-/-}$and $30 \mathrm{p53}^{+/+}$SKH-1 mice to $180 \mathrm{~mJ} / \mathrm{cm}^{2} \mathrm{UVB}(280-320 \mathrm{~nm}$; $75-80 \%$ of total energy) twice/week for 20 weeks. This irradiation induced skin tumors in $100 \%$ of irradiated mice. At the end of the experiment, tumor-bearing $p 53^{-/-}$or $p 53^{+/+}$ SKH-1 mice were randomly divided into two groups of 15 each for PDCD2 modulation. Specifically, pCDNA3.1-PDCD2 carrying PDCD2 cDNA was injected into the tail vein of each mouse. Two injections were administered at 8 a.m. and 8 p.m. for 3 days. Tumor number was recorded once every 2 weeks and plotted against time (in weeks). At the end of 25 weeks, the experiment was terminated. All of the animals were sacrificed, and skin and tumor samples were harvested.

Immunofluorescence staining of p53 and PDCD2 proteins. To assess expression of p53 and PDCD2 proteins in mouse tissues, the double-immunofluorescence technique with specific antibodies against p53 (rabbit IgG, sc-6243, Santa Cruz Biotechnology) and PDCD2 (mouse IgG1, sc-377250, Santa Cruz Biotechnology) was performed. Anti-rabbit AlexaFluor $^{\circledR} 488$ IgG or anti-mouse AlexaFluor ${ }^{\circledR} 594$ IgG
(Invitrogen) was used as the secondary antibody. Photographic images were captured using an Olympus CX71 fluorescence microscope (Olympus).

Terminal deoxynucleotidyl transferase-mediated nick end labeling (TUNEL). The TUNEL assay was performed using a kit from Roche Applied Science (no. 1684795; Indianapolis, IN, USA) according to the manufacturer's protocol. Briefly, 6- $\mu \mathrm{m}$ frozen tissue sections were rinsed three times in PBS and were incubated in $0.3 \%$ Triton X-100 (v/v) in 0.01 M PBS (pH 7.4) for $20 \mathrm{~min}$ at room temperature. Subsequently, the TUNEL reaction mixture was applied for $60 \mathrm{~min}$ at $37^{\circ} \mathrm{C}$. The fluorescence signal was detected with an Olympus microscope (model CX71) at excitation/emission wavelengths of 492/520 nm.

Statistical analysis. Statistical analysis was performed using a one-tailed Student's t-test (unilateral and unpaired). Kaplan-Meier survival plots were generated, and comparisons between survival curves were assessed using the log-rank statistical analysis. Data were analyzed using GraphPad Prism 5 software (San Diego, CA, USA), and a P-value $<0.05$ was considered to indicate a statistical significant difference.

\section{Results}

Reduced PDCD2 expression in gastric cancer tissue specimens is associated with poor survival of patients. In the present study, we collected 34 tissue specimens from gastric cancer patients and analyzed PDCD2 expression. The western blotting data showed that PDCD2 protein expression was significantly lower in the tumor tissues than that in the adjacent normal mucosa. We then analyzed expression of p53 protein and similar data were obtained ( $\mathrm{P}<0.05$; Fig. 1A). qRT-PCR data confirmed the western blotting data $(\mathrm{P}<0.05$; Fig. 1B). To assess why p53 protein was more highly expressed in normal vs. tumor tissues, we performed immunohistochemistry and found that PDCD2 protein was localized in the cytoplasm, whereas p53 protein was localized in the nuclei of cells (Fig. 1C).

We then collected follow-up data from these patients and examined the association of PDCD2 expression with patient survival. Specifically, the 34 patients were followed up between 1 month and 5 years with a median time of 28 months. Kaplan-Meier analysis showed that expression of PDCD2 protein was associated with favorable prognosis, whereas p53 expression was associated with poor prognosis of the gastric cancer patients $(\mathrm{P}<0.05$; Fig. 1D).

Antitumor effects of PDCD2 expression in gastric cancer cells is dependent on p53 expression in vitro. We next investigated the effects of exogenous PDCD2 expression in gastric cancer cell lines by transfecting PDCD2 cDNA into MKN28 and MKN45 cells. qRT-PCR and western blotting data confirmed the exogenous expression of PDCD2 in MKN28 and MKN45 cells after transfection (Fig. 2A and B). Similarly, we transfected p53 cDNA and shRNA into these two cell lines, respectively, and qRT-PCR and western blotting data confirmed p53 expression in MKN28 cells and p53 knockdown in MKN45 cells (Fig. 2C and D). 
A

$\begin{array}{llllllllllll}1 \mathrm{~N} & 1 \mathrm{C} & 2 \mathrm{~N} & 2 \mathrm{C} & 3 \mathrm{~N} & 3 \mathrm{C} & 4 \mathrm{~N} & 4 \mathrm{C} & 5 \mathrm{~N} & 5 \mathrm{C} & 6 \mathrm{~N} & 6 \mathrm{C}\end{array}$
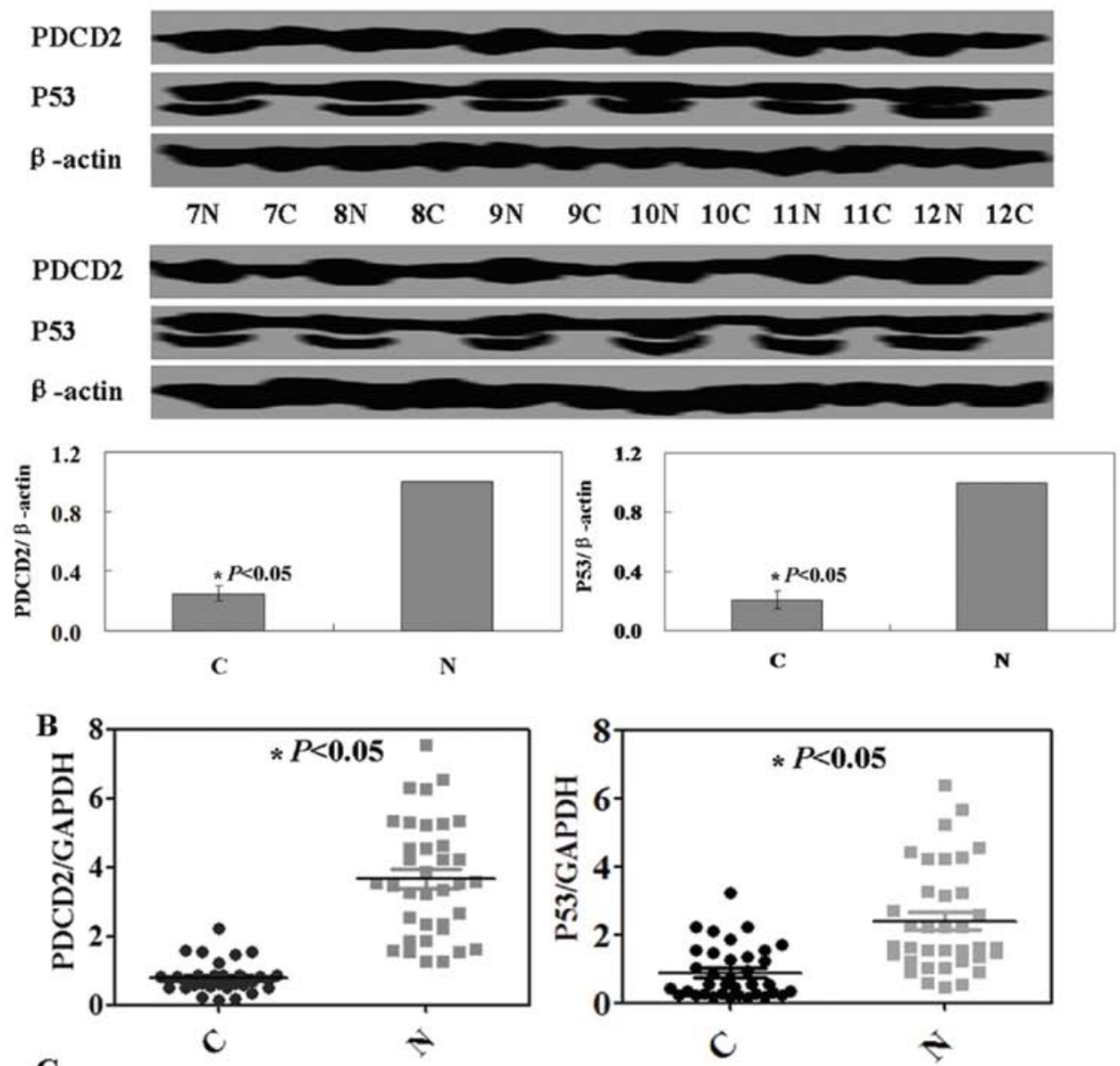

$\mathrm{C}$
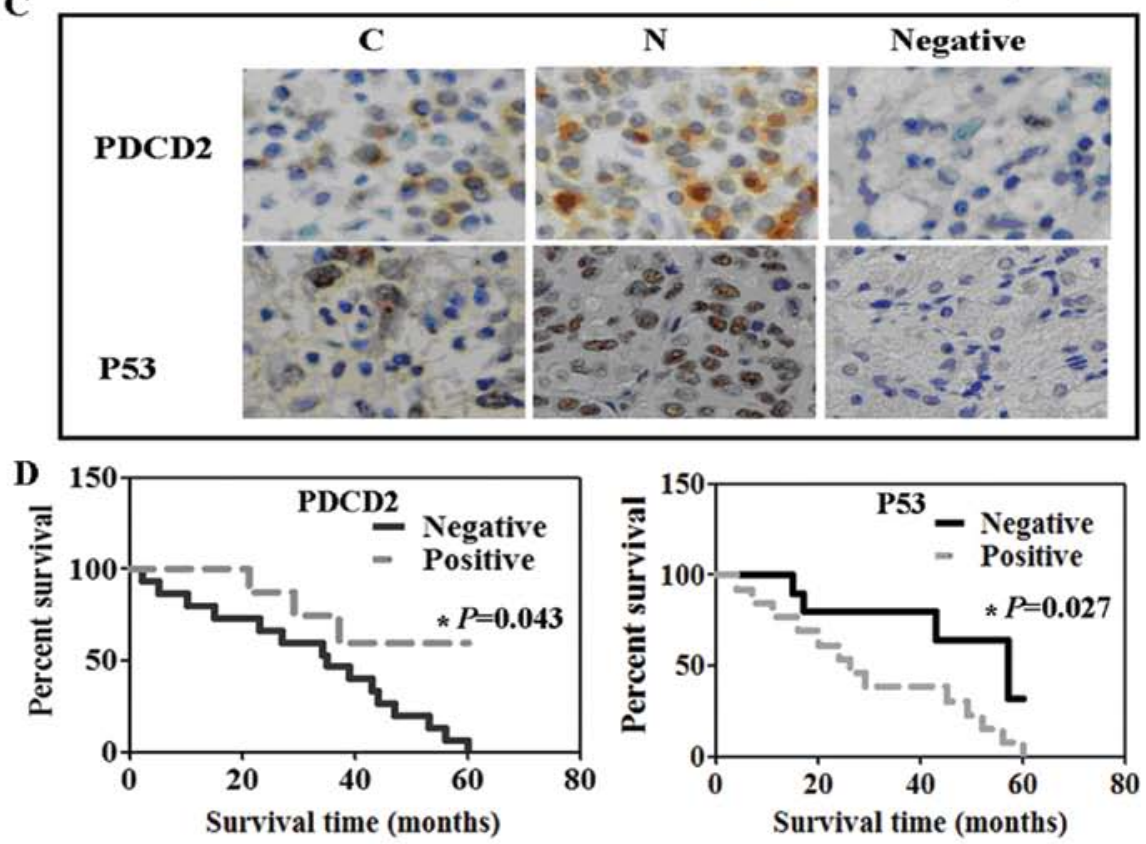

Figure 1. Differential expression of $P D C D 2 \mathrm{mRNA}$ and protein in gastric cancer tissue specimens. (A) Western blotting of PDCD2 and p53 expression. $\beta$-actin was used as an internal loading control. (B) qRT-PCR analysis of PDCD2 and p53 mRNA. GAPDH was used as an internal control. (C) Immunohistochemical analysis of PDCD2 and p53 expression. The nuclei were counterstained with hematoxylin. (D) Kaplan-Meier curves of the cumulative survival rate of patients with gastric cancer based on PDCD2 or p53 expression. N, normal; C, cancer; PDCD2, programmed cell death 2 protein.

We then assessed phenotypic changes in these cells and found slower growth in the PDCD2-expressing MKN45 cells when compared with the growth of the untreated cells by using an $\left[{ }^{3} \mathrm{H}\right]$ thymidine incorporation assay $(\mathrm{P}<0.05$; Fig. 3A). In addition, the cells showed a high level of apoptosis after PDCD2 cDNA transfection, as evidenced by Annexin V/PI double 

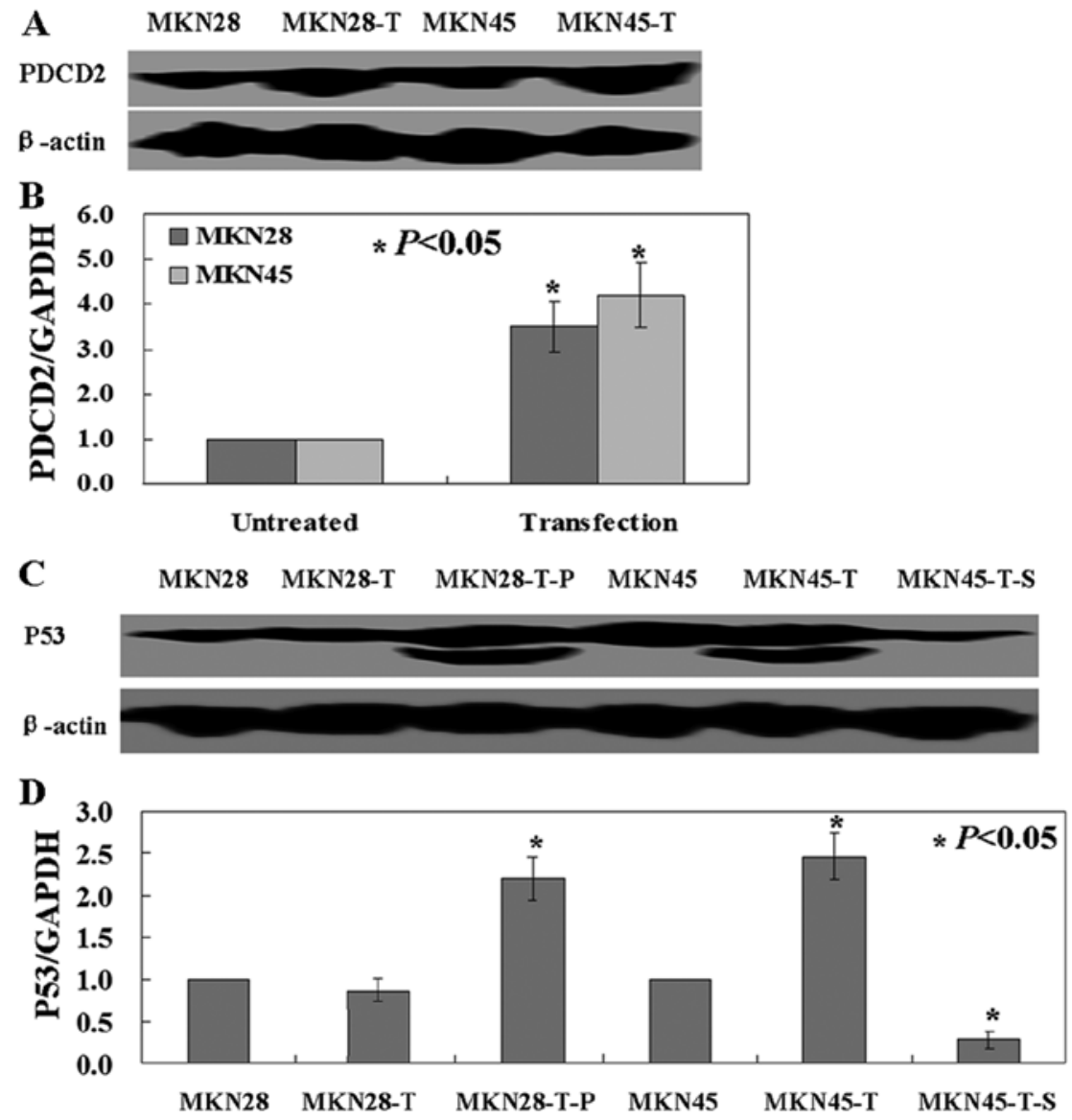

Figure 2. Expression of PDCD2 and p53 in MKN45 and MKN28 cells. (A and B) qRT-PCR and western blotting analyses of PDCD2 mRNA and protein in MKN45 and MKN28 cells transfected with pcDNA3.1-PDCD2. (C and D) qRT-PCR and western blotting analysis of $p 53$ mRNA and protein levels in MKN45 cells transfected with p53 shRNA and pcDNA3.1-PDCD2, and MKN28 cells transfected with pcDNA3.1-p53 and pcDNA3.1-PDCD2. MKN28, parental MKN28 cells; MKN28-T, MKN28 cells transfected with pcDNA3.1-PDCD2; MKN28-T-P, MKN28 cells transfected with pcDNA3.1-p53 and pcDNA3.1PDCD2; MKN45, parental MKN45 cells; MKN45-T, MKN45 cells transfected with pcDNA3.1-PDCD2; MKN45-T-S, MKN28 cells transfected with p53 shRNA and pcDNA3.1-PDCD2. PDCD2, programmed cell death 2 protein.

staining $(\mathrm{P}<0.05$, Fig. 3B). PI staining of the cells revealed that PDCD2-expressing MKN45 cells were arrested in the S phase of the cell cycle ( $\mathrm{P}<0.05$, Fig. $3 \mathrm{C})$, and $\mathrm{CldU}$ and IdU staining confirmed that the MKN45 cells were arrested at the early S phase (Fig. 3D). However, there was no effect of PDCD2 expression on the regulation of cell growth, apoptosis and cell cycle arrest in MKN45 cells transfected with $p 53$ shRNA or parental cells (Fig. 3). Similarly, PDCD2 expression was able to inhibit proliferation, but induced apoptosis and early S phase arrest in p53-overexpressing MKN28 cells (Fig. 3). These results indicate that the antitumor activities of PDCD2 in gastric cancer cells are p53-dependent.

Effects of PDCD2 expression on the regulation of UVB-induced skin carcinogenesis in $p 53^{-/-}$and $p 53^{+/+}$nude mice. To determine whether p53 is required for the antitumor effects of PDCD2, we utilized $p 53^{+/+}$and $p 53^{-/}$nude mice and induced skin carcinogenesis with UVB irradiation. Expression and localization of $\mathrm{p} 53$ and PDCD2 proteins were assessed using immunofluorescence. The data revealed that PDCD2 protein was expressed in the skin tissues of both the $p 53^{-/-}$and $p 53^{+/+}$nude mice after tail vein injection of pCDNA3.1-PDCD2 (Fig. 4A). In contrast, there was no p53 expression observed in the $p 53^{-/}$nude mice (Fig. 4A). After establishing UVB-induced skin tumorigenesis, PDCD2 expression resulted in a significant reduction in tumor number in the $p 53^{+/+}$nude mice. In contrast, there was no change in tumor number in the $p 53^{-/}$ nude mice $(\mathrm{P}<0.05$; Fig. 4B). Furthermore, we also found that PDCD2-transfected $p 53^{+++}$mice had a prolonged survival rate when compared with the survival of the PDCD2-transfected $p 53^{-/-}$mice $(\mathrm{P}<0.05$; Fig. $4 \mathrm{C})$. In addition, TUNEL staining of skin tissues showed that PDCD2 expression induced apoptosis in UVB-induced skin carcinoma of the $p 53^{+/+}$mice but not in the $p 53^{-/}$mice (Fig. 4D).

PDCD2 expression inhibits activity of the ATM/Chk1/2/p53 signaling pathway. To explore the underlying mechanism responsible for PDCD2-induced apoptosis and cell cycle arrest in gastric cancer cells, we assessed the expression of cell cycle checkpoint proteins, including the protein kinase ATM and checkpoint kinase 1 and 2 (Chk1/Chk2), which are major components of the mechanisms that oversee the control of DNA replication and genomic integrity (10). We found that levels of Chk1 and Chk 2 were not significantly changed in the MKN28 and MKN45 cells after PDCD2 transfection (Fig. 5). However, the levels of p-Chk1 and p-Chk2 were significantly increased in the MKN45 cells after PDCD2 transfection and in MKN28 cells after transfection with PDCD2 and p53 (Fig. 5). 


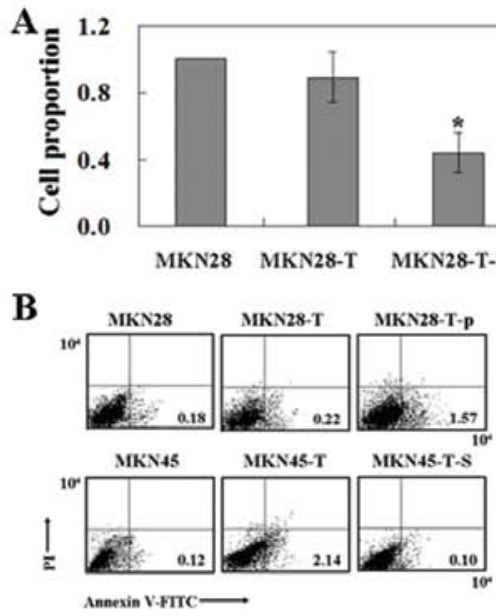

C
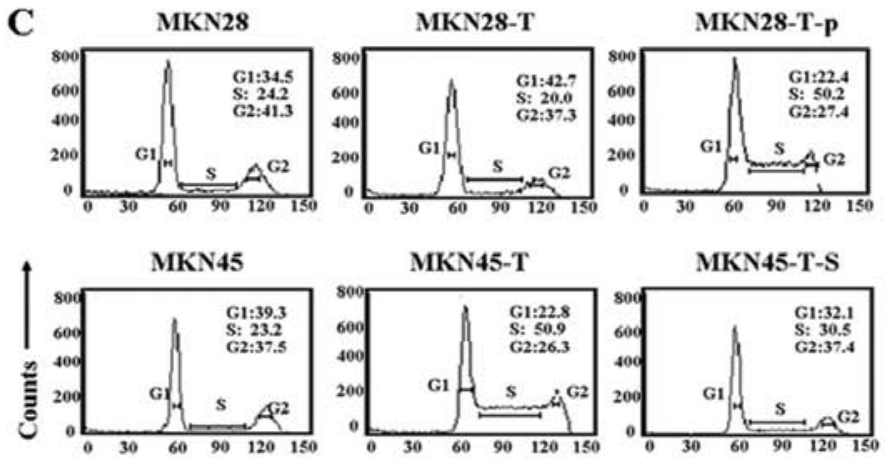

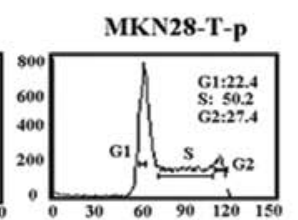

D

MKN28

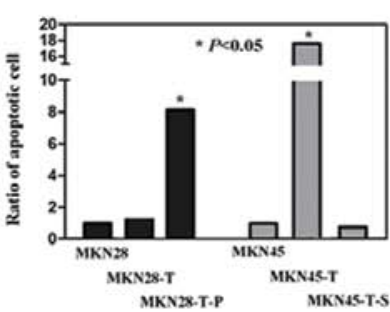

MKN45

MKN45-T

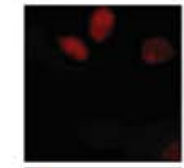

MKN45-T-S

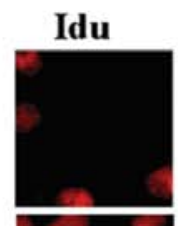

MKN28-T

MKN28-T-P
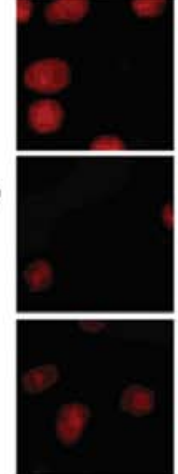
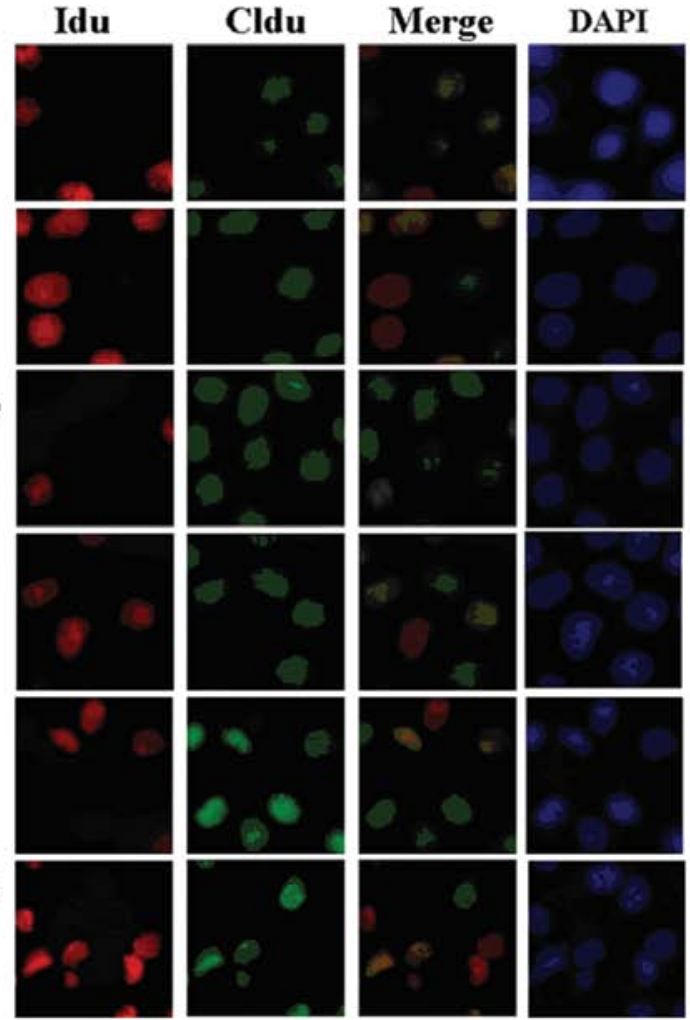

Figure 3. Effects of the antitumor activity of PDCD2 on gastric cancer cell lines in vitro. (A) (Methyl- $\left.{ }^{3} \mathrm{H}\right)$ thymidine incorporation assays. (B) Flow cytometric apoptosis assay. (C) Flow cytometric cell cycle assay. (D) Thymidine analog labeling assay. Representative images show that cells were labeled with IdU (AlexaFluor 488) only, CldU (AlexaFluor 633) only, both, or neither. PDCD2, programmed cell death 2 protein. MKN28, parental MKN28 cells; MKN28-T, MKN28 cells transfected with pcDNA3.1-PDCD2; MKN28-T-P, MKN28 cells transfected with pcDNA3.1-p53 and pcDNA3.1-PDCD2; MKN45, parental MKN45 cells; MKN45-T, MKN45 cells transfected with pcDNA3.1-PDCD2; MKN45-T-S, MKN28 cells transfected with p53 shRNA and pcDNA3.1-PDCD2. PDCD2, programmed cell death 2 protein.

Furthermore, we found that the levels of ATM, the upstream protein of Chk1 and Chk2, were also increased (Fig. 5).

\section{Discussion}

PDCD2 protein plays a role in embryonic development and tissue remodeling by inducing apoptosis, and altered PDCD2 expression could contribute to the development of human cancers. Indeed, in the present study, we detected reduced levels of PDCD2 mRNA and protein in gastric cancer tissues when compared to these levels in paired normal tissues. Our study is the first report to show the loss of PDCD2 expression in gastric cancer tissues. Previous studies have mainly focused on the role of PDCD2 in hematological tumors. Baron et al (12) showed that PDCD2 expression induces apoptosis of human erythroleukemia cells via activation of caspases. Barboza et al (13) reported that knockdown of PDCD2 expression in two different cancer cell lines (leukemia Jurkat cells and lung cancer A549 cells) significantly impairs cell proliferation and cell progression to $S$ phase of the cell cycle. In the present study, we confirmed that PDCD2 expression induced apoptosis and $\mathrm{S}$ phase arrest in p53-expressing gastric cancer cells. Furthermore, our data showed that p53 knockdown blocked the effects of PDCD2 on gastric cancer cells, but p53 activity was enhanced in PDCD2-transfected gastric cancer cells compared with the control cells.

However, our ex vivo data on p53 expression in gastric cancer tissues vs. paired normal mucosa conflict with our present knowledge on p53 protein in cells, i.e., the half-life of p53 protein is usually short and immunohistochemistry would not be able to detect p53 expression in normal tissues. However, certain p53 mutations will prolong the half-life of p53 protein and thus, immunohistochemistry can easily detect p53 expression in cells (14). In our present study, we showed that normal gastric mucosa expressed more p53 protein than did gastric cancer tissue. One explanation could be 'the field carcinogenesis' and the adjacent normal mucosae are not 'real' normal cells and that 'the field carcinogenesis' has altered gene expression and is committed to tumorigenesis (15). Indeed, gastric cancer development is usually involved in premalignant diseases, such as dysplasia or chronic gastritis and $H$. pylori infection is a single most important risk factor in developing gastric cancer $(16,17)$. In this context, the adjacent normal tissue may not be really 'normal'. Furthermore, our present data revealed that PDCD2 expression was associated with prolonged survival of patients with gastric cancer, whereas p53 expression was associated with poor prognosis of gastric cancer. The latter data were consistent with those 

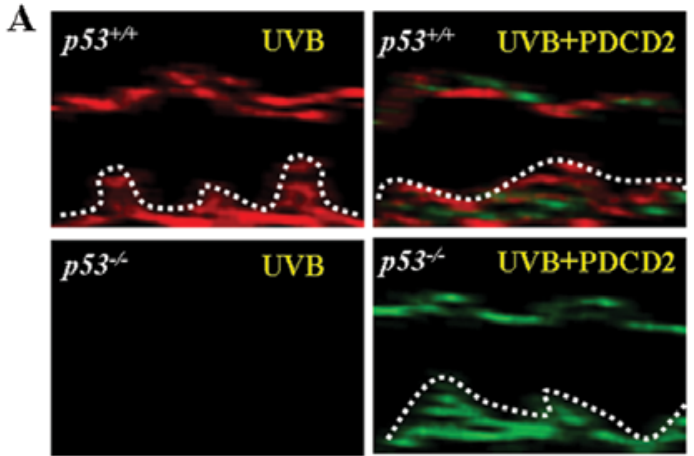

D
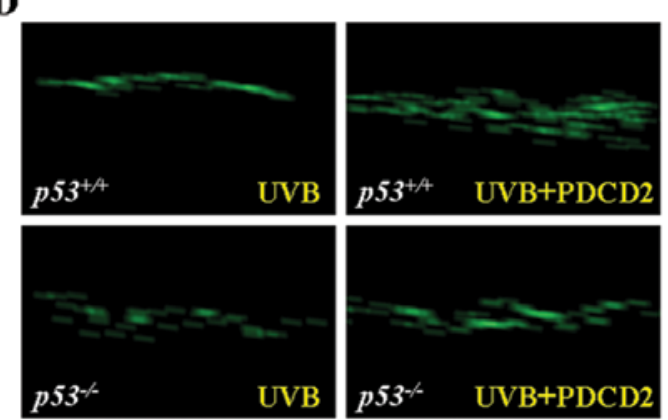
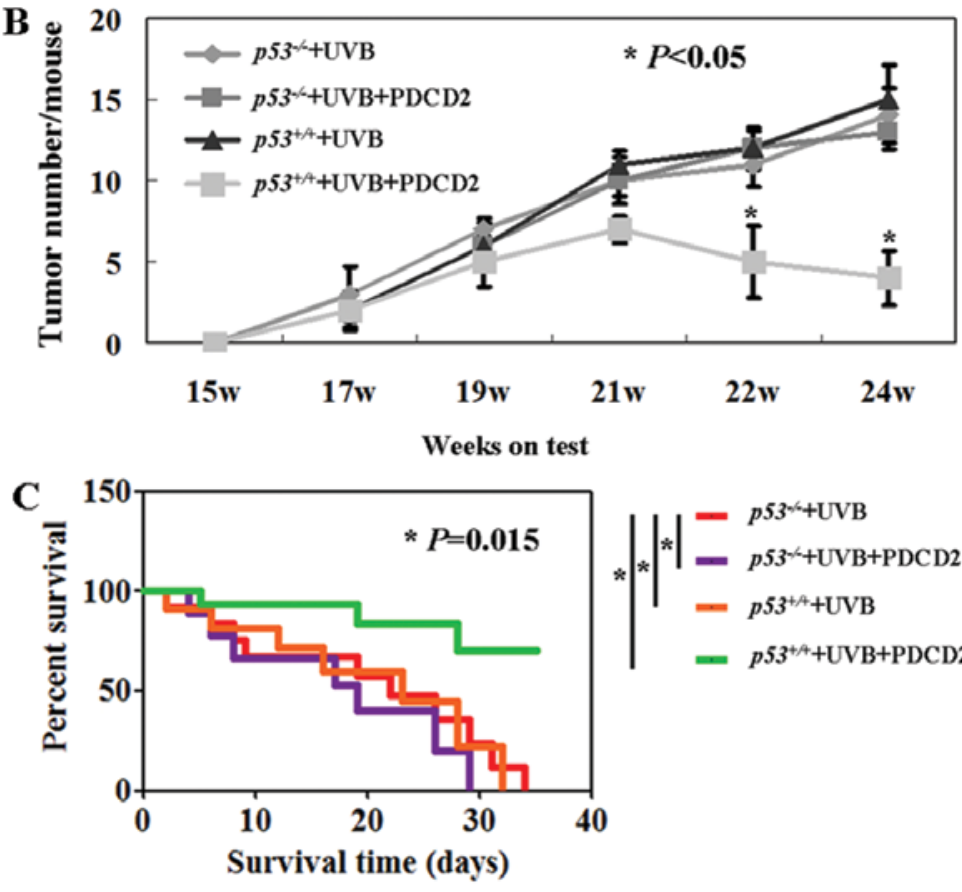

Figure 4. Effects of PDCD2 expression on the regulation of UVB-induced skin carcinogenesis in p53-knockout mice. $p 53^{-/-}$and $p 53^{+/+}$SKH-1 mice were irradiated with UVB $\left(180 \mathrm{~mJ} / \mathrm{cm}^{2}\right.$ twice/week) for 20 weeks. The mice were then injected with pCDNA3.1-PDCD2 in the tail vein. At the end of 25 weeks, the experiment was terminated, and all of the animals were sacrificed and skin and tumor samples were harvested. (A) Immunofluorescence staining of p53 and PDCD2 proteins in skin tissues. (B) Tumor growth curves in terms of tumor numbers. (C) Kaplan-Meier curves of the cumulative survival rate of mice in each group. (D) TUNEL assay revealed that PDCD2 induced apoptosis in UVB-induced skin cancer of $p 53^{-/}$and $p 53^{+/+}$mice. PDCD2, programmed cell death 2 protein.

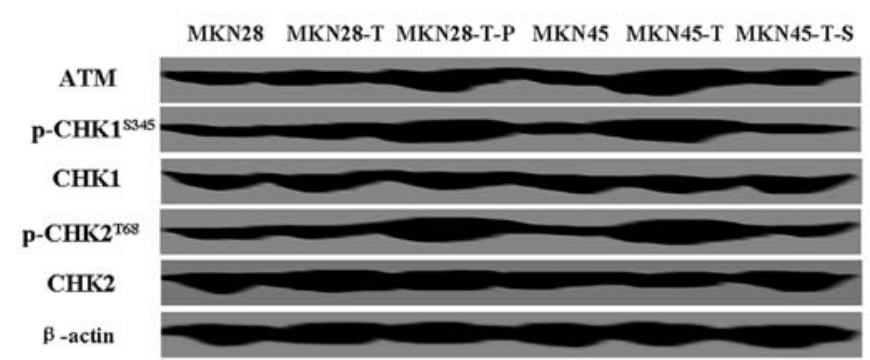

Figure 5. Changes in the activity of the ATM/Chk1/2/p53 signaling pathway in gastric cancer cells after PDCD2 and p53 modulation. PDCD2 expression induced overexpression of ATM, p-Chk1 and p-Chk2 in the presence of p53 expression, whereas Chk1 and Chk 2 showed no changes. $\beta$-actin was used as an internal loading control. PDCD2, programmed cell death 2 protein.

of previous literature reports $(18,19)$. The former data are novel and have not been reported by any study in the present PubMed database.

Moreover, we further investigated the role of p53 in mediating the effects of PDCD2 on cells by producing a mouse model of UVB-induced skin carcinogenesis in $p 53^{-/-}$and $p 53^{+/+}$nude mice. Our data showed that PDCD2 expression inhibited UVB-induced skin carcinogenesis and tumor growth by inducing apoptosis in skin cancer cells in $p 53^{+/+}$nude mice, but this did not occur in the $p 53^{-/-}$nude mice. Taken together, our in vivo and in vitro data indicate that the antitumor activities of PDCD2 are dependent on p53 expression.

In addition, we showed that activity of the ATM/Chk2/p53 pathway also mediated the antitumor effects of PDCD2 in the regulation of gastric cancer cell apoptosis and $\mathrm{S}$ phase arrest. There are four cell cycle checkpoints, i.e., the $G_{1}, G_{2} / M$, intra-S and replication checkpoints during DNA damage $(20,21)$. If DNA damage is beyond repair, then the cells will commit to apoptosis through induction of apoptosis pathways (22). ATM kinase could transduce DNA damage signals to checkpoint proteins. Activation of ATM induces phosphorylation and activation of downstream targets, including Chk1 and Chk2 (23); thus, both Chk1 and Chk2 play important roles in the DNA damage response and in cell cycle checkpoints, including the $\mathrm{S}$ phase checkpoint $(24,25)$. The present study provides evidence that PDCD2 expression induces expression of ATM, p-Chk1 and $\mathrm{p}-\mathrm{Chk} 2$ proteins and $\mathrm{S}$ phase arrest and apoptosis in gastric cancer cells that express $\mathrm{p} 53$, although a previous study provides compelling evidence supporting the role of PDCD2 in cell differentiation. For example, Kokorina et al (26) demonstrated that PDCD2 knockdown regulates the stemness of hematopoietic cells via controlling the expression of two hematopoietic progenitor cell markers, c-MYB and GATA-2, whereas loss of PDCD2 function(s) in zebra fish perturbed hematopoietic stem cell differentiation due to mitotic defects during cell cycle progression and p53-independent apoptosis (27). Scarr and Sharp (28) found that PDCD2 is a negative regulator of host cell factor-1 in a hamster cell line. These studies on PDCD2 were all conducted in normal cells, but only a few reports have shown the role of PDCD2 in cancer cells. Baron et al (12) indeed revealed that PDCD2 expression induced the apoptosis of Burkitt lymphoma cells by activation of caspases. However, to date, there has been no report showing that the antitumor activity of PDCD2 is p53 dependent. However, it remains to 
be determined whether loss of PDCD2 expression impacts the ability of p53 to induce apoptosis in cancer cells. Furthermore, p53 may not be the only protein with which PDCD2 associates and thus, a number of pathways may need to be altered after loss of PDCD2 expression.

In conclusion, our present study provides proof-of-principle data, and future studies will explore whether PDCD2 can be used as a biomarker to predict the outcomes of gastric cancer patients and whether the target of PDCD2 can be used as a novel strategy in the treatment of gastric cancer patients.

\section{Acknowledgements}

This study was supported in part by grants from the Science and Technology Plan for Social Development of the Zhejiang Provincial Science Department (2014C33236).

\section{References}

1. Jemal A, Bray F, Center MM, Ferlay J, Ward E and Forman D: Global cancer statistics. CA Cancer J Clin 61: 69-90, 2011.

2. Tahara E: Genetic pathways of two types of gastric cancer. IARC Sci Publ 157: 327-349, 2004.

3. Owens GP, Hahn WE and Cohen JJ: Identification of mRNAs associated with programmed cell death in immature thymocytes Mol Cell Biol 11: 4177-4188, 1991.

4. Kawakami T, Furukawa Y, Sudo K, et al: Isolation and mapping of a human gene (PDCD2) that is highly homologous to Rp8, a rat gene associated with programmed cell death. Cytogenet Cell Genet 71: 41-43, 1995.

5. Merup M, Moreno TC, Heyman M, et al: 6q deletions in acute lymphoblastic leukemia and non-Hodgkin's lymphomas. Blood 91: 3397-3400, 1998.

6. Minakhina S, Druzhinina M and Steward R: Zfrp8, the Drosophila ortholog of $P D C D 2$, functions in lymph gland development and controls cell proliferation. Development 134: 2387-2396, 2007.

7. $\mathrm{Mu} \mathrm{W}$, Munroe RJ, Barker AK and Schimenti JC: PDCD2 is essential for inner cell mass development and embryonic stem cell maintenance. Dev Biol 347: 279-288, 2010.

8. Ramalho-Santos M, Yoon S, Matsuzaki Y, Mulligan RC and Melton DA: 'Stemness': transcriptional profiling of embryonic and adult stem cells. Science 298: 597-600, 2002.

9. Skottman H, Mikkola M, Lundin K, et al: Gene expression signatures of seven individual human embryonic stem cell lines. Stem Cells 23: 1343-1356, 2005.

10. Fan CW, Chan CC, Chao CC, Fan HA, Sheu DL and Chan EC: Expression patterns of cell cycle and apoptosis-related genes in a multidrug-resistant human colon carcinoma cell line. Scand J Gastroenterol 39: 464-469, 2004.

11. Lou Y, Peng Q, Nolan B, Wagner GC and Lu Y: Oral administration of caffeine during voluntary exercise markedly decreases tissue fat and stimulates apoptosis and cyclin B1 in UVB-treated skin of hairless p53-knockout mice. Carcinogenesis 31: 671-678, 2010 .
12. Baron BW, Hyjek E, Gladstone B, Thirman MJ and Baron JM: PDCD2, a protein whose expression is repressed by BCL6, induces apoptosis in human cells by activation of the caspase cascade. Blood Cells Mol Dis 45: 169-175, 2010.

13. Barboza N, Minakhina S, Medina DJ, et al: PDCD2 functions in cancer cell proliferation and predicts relapsed leukemia. Cancer Biol Ther 14: 546-555, 2013.

14. Andreotti V, Ciribilli Y, Monti P, et al: p53 transactivation and the impact of mutations, cofactors and small molecules using a simplified yeast-based screening system. PLoS One 6: e20643, 2011.

15. Yellapa A, Bitterman P, Sharma S, et al: Interleukin 16 expression changes in association with ovarian malignant transformation. Am J Obstet Gynecol 210: 272.e1-10, 2014.

16. Everett SM, White KL,Drake IM, et al: The effect of Helicobacter pylori infection on levels of DNA damage in gastric epithelial cells. Helicobacter 7: 271-280, 2002.

17. Canzian F, Franceschi S, Plummer M, et al: Genetic polymorphisms in mediators of inflammation and gastric precancerous lesions. Eur J Cancer Prev 17: 178-183, 2008.

18. Lazăr D, Tăban S, Sporea I, et al: The immunohistochemical expression of the p53-protein in gastric carcinomas. Correlation with clinicopathological factors and survival of patients. Rom J Morphol Embryol 51: 249-257, 2010.

19. Liu X, Wang S, Xia X, et al: Synergistic role between 553 and JWA: prognostic and predictive biomarkers in gastric cancer. PLoS One 7: e52348, 2012.

20. Harrison JC and Haber JE: Surviving the breakup: the DNA damage checkpoint. Annu Rev Genet 40: 209-235, 2006.

21. Sancar A, Lindsey-Boltz LA, Unsal-Kaçmaz K and Linn S: Molecular mechanisms of mammalian DNA repair and the DNA damage checkpoints. Annu Rev Biochem 73: 39-85, 2004.

22. van Jaarsveld MT, Wouters MD, Boersma AW, et al: DNA damage responsive microRNAs misexpressed in human cancer modulate therapy sensitivity. Mol Oncol 8: 458-468, 2014.

23. Smith GC, Cary RB, Lakin ND, et al: Purification and DNA binding properties of the ataxia-telangiectasia gene product ATM. Proc Natl Acad Sci USA 96: 11134-11139, 1999.

24. Stracker TH, Usui T and Petrini JH: Taking the time to make important decisions: the checkpoint effector kinases Chk1 and Chk2 and the DNA damage response. DNA Repair (Amst) 8: 1047-1054, 2009.

25. Sun X, Liu B, Wang J, Li J and Ji WY: Inhibition of p21-activated kinase 4 expression suppresses the proliferation of Hep-2 laryngeal carcinoma cells via activation of the ATM/Chk1/2/p53 pathway. Int J Oncol 42: 683-689, 2013.

26. Kokorina NA, Granier CJ, Zakharkin SO, Davis S, Rabson AB and Sabaawy HE: PDCD2 knockdown inhibits erythroid but not megakaryocytic lineage differentiation of human hematopoietic stem/progenitor cells. Exp Hematol 40: 1028-1042.e3, 2012.

27. Kramer J, Granier CJ, Davis S, et al: PDCD2 controls hematopoietic stem cell differentiation during development. Stem Cells Dev 22: 58-72, 2013

28. Scarr RB and Sharp PA: PDCD2 is a negative regulator of HCF-1 (C1). Oncogene 21: 5245-5254, 2002. 